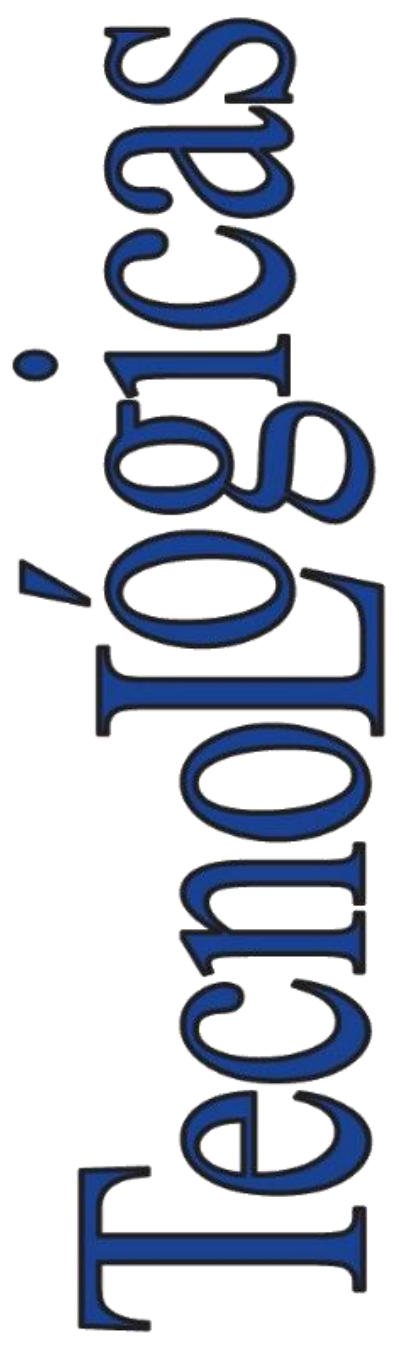

ISSN-p: 0123-7799 ISSN-e: $2256-5337$

Vol. 24, nro. 50, e1683

Recibido: 2 junio 2020 Aceptado: 8 septiembre 2020 Disponible: 10 noviembre 2020

CInstituto Tecnológico Metropolitano Este trabajo está licenciado bajo una Licencia Internacional Creative Commons Atribución (CC BY-NC-SA)

\section{Retos y oportunidades para la industria minera como potencial impulsor del desarrollo en Colombia}

\section{Challenges and Opportunities for the Mining Industry as a Potential Driver of Development in Colombia}

\author{
(D) OJuan D. Ospina-Correa ${ }^{1}$; \\ (iD)Juan G. Osorio-Cachaya ${ }^{2}$; \\ (D) Ángela M. Henao-Arroyave ${ }^{3}$; \\ (DDiego A. Palacio-Acevedo4; \\ (D) Jim Giraldo-Builes ${ }^{5}$
}

${ }^{1}$ Universidad Autónoma Latinoamericana, Medellín-Colombia, juan.osinaco@unaula.edu.co 2Universidad Nacional de Colombia, Medellín-Colombia, jgosorioc@unal.edu.co

${ }^{3}$ Universidad Nacional de Colombia, Medellín-Colombia, amhenaoa@unal.edu.co ${ }^{4}$ Servicios Hidrogeológicos Integrales S.A.S, Medellín-Colombia, diego.palacio@shi-colombia.com 5Institución Universitaria Pascual Bravo, Medellín-Colombia, jim.giraldo@pascualbravo.edu.co

Cómo citar / How to cite

J. D. Ospina-Correa; J. G. Osorio-Cachaya; Á. M. Henao-Arroyave; D. A. Palacio-Acevedo; J. Giraldo-Builes, "Retos y oportunidades para la industria minera como potencial impulsor del desarrollo en Colombia", TecnoLógicas, vol. 23, nro. 50, e1683, 2021. https://doi.org/10.22430/22565337.1683 


\section{Resumen}

Colombia ha incluido la minería en su plan estratégico de desarrollo como principal motor productivo. Surgen intereses y apuestas de inversión internacional que potencian la expansión de la minería a gran escala. El oro sigue incrementando su valor en plena tormenta bursátil por la expansión del coronavirus, donde alcanza un precio de 1940 dólares-onza, se ubica en valores máximos durante los últimos años, demuestra ser el mecanismo en la huida del riesgo de inversores, se potencia la industria minera y, a su vez, la expansión de los procesos de minería artesanal y en pequeña-escala. Las consecuencias por la expansión de las actividades de extracción mineral pueden conducir a un estado de calamidad ambiental, no sólo social, sino a ecosistemas y salud de las comunidades aledañas. En este artículo, se hace una revisión de la literatura asociada a la industria minera, destacando los principales desafíos del sector a partir de la presentación de una mirada integral y multidisciplinar del entorno, resaltando algunos indicadores ambientales y su impacto en el ámbito local. Esto permite sugerir, desde la perspectiva académica, las bases mínimas para implementar mejores prácticas de política ambiental para desarrollar una producción minera sostenible. Para esto, se establecen los puntos críticos para la gestión normativa y la evaluación de la sostenibilidad, estrategia fundamental para la toma de decisiones en el sector. Estos puntos críticos permiten priorizar el desarrollo de métricas de eficiencia en el proceso de transformación mineral y, de esta manera, aportar a la construcción de indicadores comunes de protección de la biodiversidad. Desde el punto de vista social, la gestión integral permitirá fortalecer mejores prácticas y apelará por la responsabilidad social-corporativa, otorgándoles a las comunidades locales los mecanismos de participación necesarios para intervenir en los conflictos por las áreas mineras.

\section{Palabras clave}

Sostenibilidad ambiental, tendencias sociales, sostenibilidad y minería de oro, cuantificación de la sostenibilidad ambiental, sostenibilidad ambiental y de recursos.

\section{Abstract}

Mining has been included as the main driver of productive development in Colombia's strategic development plan. In particular, the expansion of large-scale mining has been driven by emerging foreign investment interests and projects. The price of gold continues to rise, amidst the stock market crash caused by the coronavirus outbreak, reaching 1940 USD per ounce. In fact, gold has reached its highest values in recent years, thus proving to be the mechanism for investors to avoid risk. Also, it has boosted the mining industry, as well as artisanal and small-scale mining. However, the consequences of the expansion of mineral extraction activities could lead to a state of environmental emergency, affecting not only the social sphere but also the ecosystems and the health of the surrounding communities. In this paper, we provide a literature review on the mining industry. In addition, we highlight its main challenges from a comprehensive and multidisciplinary contextual perspective that involves outlining some environmental indicators and their impact at the local level. This allows us to suggest, from an academic standpoint, the minimum bases required to implement best practices in environmental policy and, thus, achieve a more sustainable mining production. For this purpose, we define the critical factors for regulatory management and sustainability assessment-a fundamental strategy for decision-making in the sector. These critical points make it possible to prioritize the development of efficiency metrics in mineral transformation and contribute to the construction of common indicators for the protection of biodiversity. From a social point of view, a comprehensive management will strengthen best practices and call for corporate social responsibility to provide local communities with the necessary participation mechanisms to intervene in conflicts over mining areas.

\section{Keywords}

Environmental sustainability, social trends, sustainability and gold mining, quantification of environmental sustainability, environmental and resource sustainability. 


\section{INTRODUCCIÓN}

La era colonial en Sur América trajo una expansión de la minería de oro y plata. En Colombia, la minería empezó a establecerse en la cuenca alta del río Cauca, donde los indicios de la riqueza de los yacimientos permitían el establecimiento de campamentos para el beneficio [1]. Los depósitos se explotaban de manera rudimentaria hasta el agotamiento y, mediante amalgamación con mercurio, se recuperaban los valores metálicos, buscando con este método mejorar el rendimiento del proceso [2]. Las características geomorfológicas de estos yacimientos determinan su espacialidad y ubicación, localizándolos hacia el norte del país sobre los Andes. Estos depósitos se concentran en tres regiones: en la cuenca del río Cauca, en el valle superior del río Magdalena y en las zonas bajas de la costa pacífica [2]. En la zona nordeste del país, tanto en Antioquia como Chocó, se ha logrado durante los últimos diez años cerca del $70 \%$ de la producción nacional [3]-[5].

Actualmente, el sector de la minería del oro en Colombia está formado por tres subsectores principales: (i) minería artesanal, (ii) minería a pequeña escala y (iii) minería a gran escala [6], [7]. La minería artesanal y en pequeña escala están aumentando sus operaciones debido al incremento del valor del oro, más aún, en plena tormenta bursátil por la expansión del coronavirus, alcanzando $\approx 1900$ dólares la onza, ubicándose en máximos durante los últimos años y demostrando ser el gran ganador en la huida del riesgo de los capitales de inversión [4], [8]. La extracción artesanal, generalmente, se realiza sin asistencia técnica y de manera rudimentaria, se hace uso de mercurio, esto genera contaminación de aguas, pérdidas en la oportunidad de sustento agrario y desplazamiento de mano de obra de las comunidades, lo que conlleva a que se perciba el crecimiento del sector como una competencia desigual por el uso del agua, la tierra y sus potencialidades [9]-[11]. Se estima que en 2017, 40 millones de personas participaron directamente en operaciones mineras, en comparación con $\sim 30$ millones en 2014, 13 millones en 1999 y $\sim 6$ millones en 1993 [12]. Sólo en Colombia, el Ministerio de Minas y Energía de Colombia se ha concentrado en la formalización de $~ 300000$ mineros artesanales [13]-[16]. De acuerdo con datos del ministerio, de las 500 unidades mineras censadas, $\sim 70 \%$ no tenía permisos formales para la operación [17]. Sin embargo, la producción de oro en el país superó $\sim 60$ toneladas en 2016, donde el sector formalizado sólo representó el $13 \%$ [18], esto convierte a la minería artesanal y de pequeña escala en uno de los sectores de mayor crecimiento en Colombia en los últimos años.

En Colombia, cerca del $20 \%$ de los municipios tienen depósitos auríferos importantes, ubicándolos en el foco de inversión de empresas nacionales e internacionales a través de los marcos normativos gubernamentales que buscan que la minería sea un motor clave en el desarrollo económico del país [18]-[20]. Se estima que para el 2027 la producción de oro en Colombia crecerá a 2.3 millones de onzas, con un crecimiento promedio anual de $2.4 \%$ [21].

En este escenario, las reservas aseguran la continuación de los proyectos de exploración y extracción. En 2017, la multinacional Gran Colombia Gold logró incrementar su producción en $\sim 16 \%$, mejorando sus utilidades. En el mismo sentido, la multinacional Continental Gold apropió recursos para inversión por 389 millones de dólares con el fin de incrementar operaciones en sus plantas de procesamiento y perforación en el municipio de Buriticá [22].

Así, con el comportamiento internacional del precio del oro, platino y níquel para el periodo 2010-2020 (Figura. 1), los proyectos en minería continúan como uno de los sectores estratégicos en términos de inversión, aunque su contribución al PIB ha sido bastante limitada, $\sim 0.45 \%$ entre los años 2011-2015. Se espera que su participación aumente sustancialmente para el año 2025 [17], [23]. 


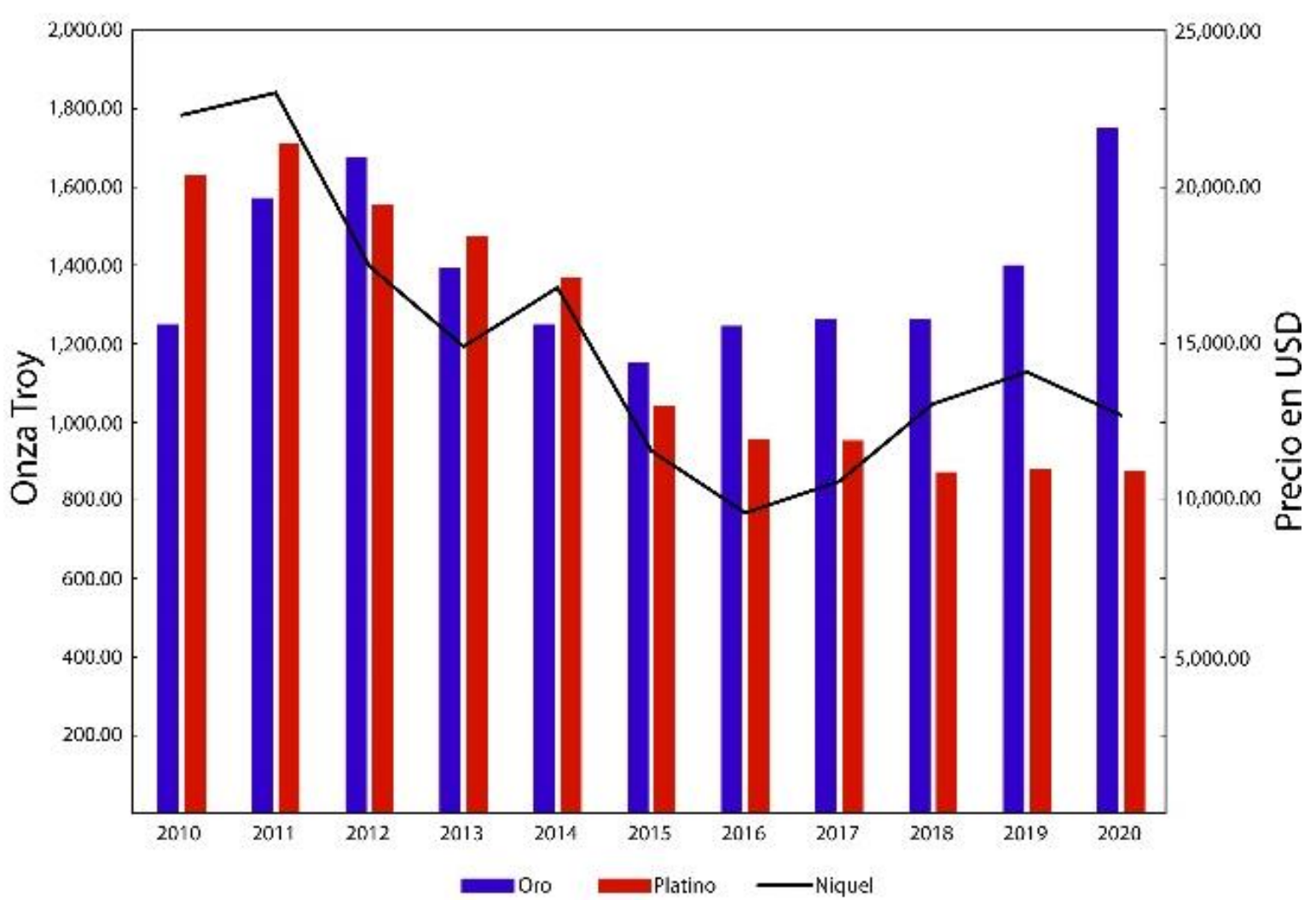

Figura. 1. Comportamiento internacional del precio del oro, platino y níquel 2010-2020. Fuente: [24]-[26].

Los indicadores económicos sugieren que la minería es un vehículo potencial para impulsar el crecimiento constante en países en desarrollo, como ha sucedido en Canadá, Estados Unidos y Australia [27]. Sin embargo, cuando el desarrollo de las actividades mineras ocurre en un contexto de instituciones sociales, políticas y económicas subdesarrolladas, la riqueza producida por la explotación de recursos no renovables tiende a ser mal utilizada, aumentando el nivel de conflicto social y generando daños dramáticos al medio ambiente, y esto en conjunto, podría dejar a una nación en desarrollo permanentemente y aún más pobre [28].

Por lo tanto, considerar el crecimiento económico del país fundamentado en industrias extractivas requiere de cuidadosas consideraciones antes de sacar conclusiones sobre los alcances y beneficios esperados, valorando los costos inherentes de la actividad. Con este panorama, los desarrollos en minería deben estar enfocados a incluir aspectos como prospección [29], formación de depósitos [30], [31], explotación y beneficio [32], perspectivas históricas [33], ambientales [34]-[36], salud pública [37], [38], ingeniería [39]-[41], aspectos culturales [42] y también derechos humanos [43]. Con acciones focalizadas en estos aspectos de interés, la industria puede desarrollar sus actividades de forma holística entre sus componentes y los actores sociales que intervienen. Sin embargo, pocos autores han tratado de unificar estas diversas dimensiones de la actividad, que permitan abordar soluciones integrales para el sector [44].

El período de estudio comprende los años entre 2000-2017, ya que el aumento en el precio del oro estimuló el surgimiento de nuevas actividades de extracción en áreas que anteriormente no eran beneficiables [45]. Esta revisión se realiza para obtener una visión general del desarrollo en las regiones mineras de oro de Colombia para la evaluación de los impactos ambientales y sociales de la actividad minera en las comunidades involucradas. 
En este contexto, esta revisión tiene como objetivo identificar las iniciativas actuales creadas por investigadores, la comunidad, el gobierno y organizaciones nacionales y/o internacionales para hacer frente a los impactos de las actividades de extracción de oro desde la perspectiva ambiental y social, además de identificar algunas oportunidades de mejora para el sector desde la perspectiva del desarrollo de comunidades de una manera ambientalmente sostenible y socialmente sólida.

\section{METOdOLOGía}

La propuesta metodológica para la elaboración de la revisión se focalizó en literatura influyente en torno al tema de estudio y se basó en un conjunto de tareas propuestas por Snyder [46] y Fink [47]. La primera actividad permite la selección de los principales interrogantes de investigación frente al tema, interrogantes que permiten localizar información para establecer métricas y comprender los desafíos, iniciativas y oportunidades que actualmente tienen acogida en el sector minero. En este sentido se definieron las siguientes premisas rectoras: $(i)$ ¿cuáles son los principales desafíos ambientales y sociales que enfrenta actualmente Colombia para el desarrollo de su industria minera? (ii) de las iniciativas actuales que se están llevando a cabo para enfrentar estos desafíos, ¿cuáles tienen seguimiento y qué tipo de seguimiento se hace? y (iii) ¿cuáles son las principales oportunidades de mejora que permitan incluir el desarrollo de comunidades que viven en áreas de influencia?

Luego de seleccionadas las premisas rectoras, se filtraron términos de búsqueda en motores y bases de datos en función de las métricas funcionales objetivo del trabajo. El proceso implicó seleccionar las publicaciones más relevantes durante el período 2000-2017, a través de Scopus, Google Scholar y Web of Knowledge. Este período temporal de selección mostró un incremento monotónico en la producción mundial de oro, que estimuló el surgimiento de nuevas actividades de extracción en áreas que anteriormente no eran rentables para la minería para poder satisfacer las demandadas crecientes del metal [48]. Para contrastar la información publicada se recurrió a información de agencias gubernamentales y organizaciones privadas, información considerada no sensible, que permitiera contrastar la realidad y tener un panorama con ambas miradas. Además, se realizó una búsqueda selectiva de acciones focalizadas en el sector. La búsqueda incluyó libros relevantes sobre el tema, así como conversaciones con diferentes expertos en minería para contrastar la información documentada. Se utilizaron los siguientes criterios:

-Tipo de estudio: los registros seleccionados deben describir detalladamente las actividades de extracción y los registros para los impactos ambientales, sociales y económicos, las métricas deben seguir un patrón claro de medición.

-Tema: los registros seleccionados deben incluir palabras clave como oro, mercurio y minería en el título y/o resumen, la localización del estudio debe tener características geomorfológicas similares a las presentadas en Colombia.

-Diseño del estudio: los artículos científicos, informes y regulaciones gubernamentales y/o privados fueron seleccionados para su inclusión en el análisis, debido a su estrecha relación con actividades de extracción pero que por la esencia misma de la entidad los datos no se presentan en publicaciones científicas.

-Año de publicación: debido a la gran actividad de nuestros legisladores, se buscó la literatura publicada entre 2000 y 2017, para poder comparar las diferentes posturas del estado frente al sector. 
-Idioma: se seleccionó la literatura inglesa, por ser una lengua prolífica en el tema, y la española, por las disposiciones administrativas de gestión.

-Estado de publicación: para la literatura científica, solo se tomó en consideración literatura revisada por pares y editoriales reconocidas en el área de la extracción de minerales. Los informes y documentos oficiales son de libre acceso desde la página web de las organizaciones y/o agencias gubernamentales.

Los resultados de la búsqueda se sintetizaron y correlacionaron de forma descriptiva mediante interpretaciones de los hallazgos basados en la experiencia de los revisores. La calidad de la revisión se controló comparando con los constructos de otras investigaciones. Finalmente, los resultados fueron presentados y discutidos con otros investigadores y profesionales para obtener comentarios que permitirán la discusión desde una perspectiva integral.

\section{RESULTADOS Y DISCUSIÓN}

\subsection{Transformación del uso del suelo y emisiones}

El oro está clasificado como uno de los activos de mejor desempeño a nivel mundial, su demanda aumentó $20 \%$ en 2016, mostrando el rendimiento más alto en casi tres décadas. Sin embargo, este crecimiento presenta retos ambientales difíciles de gestionar, debido a la generación de residuos y deterioro paisajístico [36]. En 2015, Colombia ocupó el puesto 18 entre varios países productores, con una oferta en crecimiento continuo durante los últimos 10 años, pasando de 15.6 toneladas en 2006 a 59.2 toneladas en 2015 [49]-[51], y cerca de 56000 toneladas en reservas mundiales [51]. Suponiendo que el tenor promedio de oro es $\sim 2$ partes por millón (ppm), sólo en 2015 se generaron 1500 millones de toneladas de desechos sólidos [52], [53]. Los grandes volúmenes de material fracturado, fruto de los procesos de conminución, y las piscinas excavadas para el manejo y disposición de relaves potencian la formación de corrientes de lixiviados que modifican las funciones ecológicas del suelo [54], [55]. Esto conduce a la modificación de canales, lechos y pendientes de los ríos que crean humedales artificiales o pozos inundados para suministro de agua en las operaciones, se cambia, en la mayoría de los casos, la conectividad hidrológica de humedales [54]. Según un informe publicado por la autoridad ambiental colombiana en 2016, 78939 hectáreas han sido afectadas por la extracción de oro aluvial, donde el 80 \% de las áreas se localizan en Antioquia y Chocó [56], [57]. El 8 \% de la tierra total del país afectada por sedimentos de vertederos dragados se concentra en un solo municipio: Nechí, Antioquia. Para el 2014, en el Chocó 25000 hectáreas fueron reportadas como deterioradas por malas prácticas mineras, incluyendo áreas de bosque natural, vegetación primaria y secundaria y su fauna asociada [57]. La extracción de oro también crea depósitos de relaves que se forman después de la eliminación de la capa superficial del suelo y rocas. Su magnitud depende de la tecnología minera empleada, la geología de la zona, la topografía y el clima local, estimulando la reactividad de los sitios posterior al proceso de beneficio, dando lugar a transformaciones físicas en los minerales, donde procesos químicos como la oxidación, la acidificación, la hidrólisis, la lixiviación de metales y la precipitación de sulfatos son fuentes potenciales de contaminación [58]. 


\subsection{Emisiones y sus efectos}

Las operaciones a pequeña escala representan $\sim 30 \%$ de la producción mundial de oro y $\sim 70 \%$ de la producción total en Colombia [59]. La acumulación de mineral para su posterior procesamiento en entables cercanos a centros urbanos, donde se agrega mercurio en pequeños molinos para la separación y extracción, es una práctica común [60]. Los mineros prefieren el mercurio porque es fácil de usar y transportar, ancestral, de fácil acceso y barato, también les permite realizar todo el proceso sin depender de cooperativas [61]. Según Legiscomex, en 2011, dos años antes de la entrada en vigencia de la Ley de cero Mercurio, los distribuidores de mercurio para Colombia, el cual es importado y comercializado principalmente por empresas privadas (en toneladas), eran, México (233.8), España (184.6), Países Bajos (180.3), EE.UU. (152.1), Alemania (82.1) y Perú (21.7) [62]. Dos técnicas son empleadas para la amalgamación del oro: (i) amalgamar todo el mineral acumulado luego del proceso de trituración, donde la eficiencia en la recuperación no supera el 30 \% [18] y (ii) la amalgamación en asocio con concentración gravimétrica, donde la eficiencia se incrementa $\sim 70 \%$, así como sus costos, disminuyendo la utilidad real [18]. Como parte de la transacción, los mineros deben dejar sus relaves para ser lixiviados con cianuro por los propietarios de los entables, como parte del pago por el servicio [63].

En 2011, debido a este conjunto de malas prácticas, Colombia fue clasificada como la tercera fuente de emisiones de mercurio en el mundo y el mayor contaminante de mercurio per cápita [9]. Los datos sugieren que la falta de tecnología para reducir las emisiones es uno de los principales indicadores del grado de informalidad del sector, permitiendo contaminación por metales pesados en sedimentos y humedales cercanos que actúan como sumideros [64], [65]. El complejo de humedales productivos en la depresión Momposina, entre los ríos Cauca y Magdalena, forma una de las regiones con mayor biodiversidad del mundo, amenazados por la minería, donde se han medido altas concentraciones de mercurio en los sedimentos del río Cauca [66]. Los estudios sugieren que los ambientes acuáticos y la salud humana están en riesgo debido a la biodisponibilidad de compuestos inestables de mercurio que podrían ingresar y acumularse en la cadena alimentaria. Estos niveles excesivos de riesgo a los que están expuestos los residentes de las comunidades mineras, debido a inhalación de mercurio, también afectan comunidades que generalmente son pesqueras [67]. Se ha encontrado evidencia de bio-acumulación de mercurio en seis especies de peces del río Nechí [68]. Los resultados sobre el ciclo de vida muestran que la huella ambiental de la producción de oro es mayor a la generada en procesos de extracción de cobre y níquel, en relación a las emisiones de gases, el consumo de agua y la carga de desechos sólidos [69], atribuible al bajo tenor de oro, lo que incrementa la demanda de mineral, y al uso de energía en la etapa de procesamiento.

\subsection{Retos en la sostenibilidad minera y políticas de gestión}

Los procesos de innovación y planificación para el desarrollo de mejores prácticas de política ambiental y gestión de recursos minerales para la sostenibilidad de la industria minera están motivados por preguntas fundamentales sobre las interacciones complejas y frágiles entre la naturaleza y la sociedad, así como por necesidades sociales urgentes y apremiantes [70], [71], [72]. Cada uno de los objetivos de la investigación en este campo debe ir más allá de los problemas disciplinares académicos tradicionales, ubicándose en las complejas interacciones entre las comunidades y el entorno [71]. El avance de los procesos de innovación y planificación para el desarrollo de mejores prácticas de política ambiental y su influencia en la sostenibilidad implica no transcurrir los caminos habituales de la 
investigación desarrollada, ya que convencionalmente el campo de acción se encuentra fuera del dominio de los entes que intentan asumir los procesos de fiscalización [72], entendiendo que la investigación científica y la aplicación práctica, si bien se mezclan de múltiples maneras sinérgicas, a veces lo hacen lejos de los marcos políticos locales. Con intención de la unión sinérgica entre esas dos líneas de razonamiento, a comienzos de siglo se plantearon preguntas fundamentales de investigación en mejores prácticas de políticas ambientales y sostenibilidad industrial [72], donde el consenso para el desarrollo permitió una síntesis masiva de las afectaciones y las estrategias de recuperación sobre los ecosistemas bajo análisis, buscando incrementar la capacidad para apoyar el bienestar poblacional. En este sentido, la comunidad internacional construyó un marco de análisis para la Evaluación de Ecosistemas del Milenio (EEM). En esta síntesis, se combinaron conceptos básicos y aplicados de la sostenibilidad y se desafió a la comunidad internacional a sintetizar lo que se conocía sobre la sostenibilidad de las industrias extractivas y de alto impacto en formas relevantes para la generación de regulaciones y políticas públicas.

Los resultados sugieren grandes fortalezas pero también muestran lagunas en la ciencia subyacente [73], permitiendo establecer nuevas posibilidades de medición y proyección de los efectos de las decisiones políticas y las acciones humanas sobre los ecosistemas, los servicios que ellos brindan y el bienestar de las comunidades aledañas y beneficiarias. Los desarrollos se enfocan en diversos frentes, como los servicios ecosistémicos [74], las dinámicas del uso del suelo [75], la gobernanza de los recursos de propiedad pública [28], las conexiones entre la historia del sistema y la modificación del uso de la tierra [76] y el modelado de sistemas dinámicos [77]. Al mismo tiempo, resaltando la necesidad de la comunidad política por información pertinente y veraz que permita valorar por qué los impactos de la industria están creciendo, y en este sentido, potenciar el progreso en este campo de interés colectivo, lo que está revelando nuevos desafíos en la necesidad de evaluar, proyectar y gestionar los flujos de servicios ambientales con afectación a los ecosistemas y los cambios en el bienestar poblacional. Considerando como punto de partida los principios de EEM para comprender las brechas existentes en las políticas de gestión y regulación ambiental, las prioridades de investigación, basadas en los resultados de estudios conjuntos realizados entre 2000 y 2019 avalados por el Consejo Internacional de Ciencia, la Organización de las Naciones Unidas para la Educación, la Ciencia y la Cultura y la Universidad de las Naciones Unidas sobre los esfuerzos para comprender las dinámicas de estos ecosistemas acoplados.

\subsection{Iniciativas para la recuperación de residuos y protección ambiental}

La sostenibilidad en las prácticas de recuperación son complejas, dependen no solo de la recuperación de la vegetación, sino también de la mejora en la cantidad y calidad de la materia orgánica del suelo, potenciando la restauración de procesos de ciclo de nutrientes [78], estabilización, control de contaminación y reducción de transporte de metales pesados [79].

Se obliga a una gestión para la recuperación adecuada del paisaje, la infiltración de agua y el límite de las zonas de escorrentías, evitando así la erosión del suelo [75]. Se ha documentado, por ejemplo, la existencia de proyectos de recuperación forestal en Cáceres, Antioquia, con acacia mangium y algunas especies arbóreas nativas con la ayuda de microorganismos y nutrientes, proceso con participación local de pobladores [80].

Las especies arbóreas empleadas muestran altos niveles foliares de nitrógeno y capacidad para hacer simbiosis con bacterias fijadoras de nitrógeno, aunque el nivel de fosforo biodisponible en el suelo fue el nutriente que limita la productividad [80]. En la misma línea de forestación, Mineros S.A. estableció parcelas agroforestales para recuperar depósitos en los municipios de Nechí y El Bagre, Antioquia [79]. Para el desarrollo de estas estrategias de 
protección ambiental, las empresas mineras han facilitado instalaciones básicas de almacenamiento y saneamiento, así como capacitación para producir compost y estructuras básicas para almacenar en viveros las especies seleccionadas [13]. Es imprescindible que los planes de cierre y fin de operaciones velen por la recuperación de las propiedades físicas y químicas del suelo, por su funcionalidad y el paisajismo, por la diversidad funcional del suelo, y por el secuestro de carbono y las sinergias con comunidades bacterianas. Estas medidas deben ser contempladas en conjunto con el apoyo de las comunidades locales, políticas efectivas, financiamiento y ajustes legislativos apropiados [81].

La extracción de oro requiere del monitoreo a largo plazo, junto con un plan de manejo de escorrentías para reducir los drenajes ácidos [82]. Por ejemplo, cubrir el material potencialmente formador de ácido con una capa de arcilla puede minimizar el potencial de intemperismo y oxidación dentro del sitio, reduciendo los daños a lo largo del tiempo [83]. Es en este punto donde los proyectos de recuperación a largo plazo proponen prácticas certificadas y acciones de campo, con el apoyo de comunidades locales, políticas efectivas y financiamiento. Estas, en conjunto, son la clave para la dinamización positiva de futuros proyectos extractivos [84].

\subsection{Remediación de zonas afectadas por mercurio}

Mundialmente se han desarrollado varias iniciativas para la eliminación del mercurio de los procesos mineros. La Ley de Prohibición de Exportación de Mercurio (MEBA), aprobada en Estados Unidos en 2008, limita la disponibilidad de mercurio elemental en los mercados, especialmente en el sector de la minería artesanal [85], además del Convenio de Minamata que prohíbe la producción y exportación de mercurio desde 2011 [86]. En el ámbito local, otras medidas que permitirían abordar la restricción del uso del mercurio es la aplicación de la ley que prohíbe el uso de mercurio en la extracción de oro y los acuerdos contractuales establecidos entre mineros artesanales y empresas mineras. Esto último permite a los mineros vender el material extraído a la empresa para ser procesado por cianuración [87].

Incluso, formas complementarias de intervención incorporan la creación de asociaciones locales entre mineros artesanales, relación que permite acumular el capital financiero para adquirir tecnologías de menor impacto, así como el emprendimiento asociativo y el desarrollo de esquemas de co-gestión. Sin embargo, más allá de restringir el uso de mercurio, es necesario estrategias para remediar la contaminación en el suelo y el agua. Los tratamientos fisicoquímicos permiten remediar los suelos contaminados con metales, pero suelen ser costosos y pueden afectar las propiedades del suelo, dejándolos inadecuados para el crecimiento de plantas [88].

La fitorremediación de metales pesados generalmente se realiza con especies hiperacumuladoras que tienen la capacidad de incorporar en su estructura metales hasta el $0.01 \%$ de su peso seco [89]. Experimentos en invernadero con Euphorbiaceae evaluaron la efectividad para la fito-remediación de suelos contaminados con mercurio sin translocación del mercurio a partes aéreas de la planta, lo que indica que el mercurio permanecía principalmente en las raíces [90]. En este sentido, también se ha evaluado Marantaceae, Piperaceae, y Cyperaceae: las tres especies demostraron tener la capacidad de acumular altos niveles de mercurio en sus raíces y brotes. Sin embargo, debido a las altas concentraciones de metales pesados de las plantas utilizadas para la fitorremediación, aunque uso como alimento podría representar riesgos para el ganado, la vida silvestre y seres humanos [91]. 


\subsection{Estrategias para una extracción ambientalmente sostenible}

La vida de un proyecto minero está limitada por la geología del yacimiento y por las fluctuaciones del mercado de valores, razón por la cual el sector minero debe buscar formas de reducir costos y riesgos locales, brindando beneficios locales duraderos [92]. Es imperativo que la actividad minera permita satisfacer las necesidades de las generaciones presentes y futuras, internalizando el costo de externalidades. Sin embargo, la sostenibilidad de un recurso no renovable debe considerar que la demanda mundial de materias primas va a disminuir y se necesita recurrir al reciclaje y la reutilización para hacer que la actividad económica de la minería sea sostenible [93].

Para la sostenibilidad, la eficiencia en el beneficio de los recursos es un aspecto importante en términos de reducción del impacto sobre ecosistemas y disminución de las emisiones derivadas de las actividades de procesamiento [94]. Para Colombia, la información en este sentido, sobre el uso racional del agua y la energía, se limita a los informes proporcionados por dos empresas mineras. Por tanto, es un área que las autoridades ambientales deben monitorear para poder optimizar. Es necesario implementar programas de eficiencia en el uso racional de energía y agua, así como fomentar el uso de energía solar, geotermia, hidroeléctrica, eólica y biomasa [95]. La liberación de elementos como antimonio, bismuto, plomo, mercurio, cianuro, arsénico, cromo, hierro, así como las emisiones de gases de efecto invernadero y cualquier otra sustancia que pueda tener un impacto perjudicial sobre el medio ambiente y la salud, deben ser reguladas por la legislación ambiental de manera integral, especialmente para las actividades de extracción de oro, ya que el potencial de contaminación de los cuerpos de agua, a través de descargas directas o accidentales, es muy alto. Para ello, es necesario garantizar la adopción de estándares internacionales de protección ambiental, integrando los temas de biodiversidad en las prácticas corporativas de gestión ambiental [96].

Por ejemplo, la actividad industrial en la cadena montañosa del departamento de Santander, que representa una de las mayores reservas de oro sin explotar del mundo, se enfrenta a una operación minera a gran escala: la zona rica en agua del Páramo de Santurbán incluye 11700 hectáreas de terreno en las que no se permite ninguna actividad económica [97]. En Colombia, los páramos son territorios estratégicos, los cuales estimulan sistemas de producción agrícola, y son el principal suministro de agua para muchos centros urbanos [98].

Esta política de protección del agua debe prevalecer sobre el recurso mineral de acuerdo con el POMCAS de la cuenca [99].

\subsection{Desarrollo de marcos de evaluación para la sostenibilidad}

La sostenibilidad en el entorno local debe reflejarse en valor agregado y riqueza generada por la explotación de las reservas minerales [100]. Por lo tanto, dadas las características especiales de cada proyecto, aspectos bióticos y abióticos, necesidades técnicas, impactos ambientales, capacidad industrial, magnificación de dramas sociales y económicos por la modificación cultural y laboral local, es necesario implementar un marco general para la evaluación del nivel de sostenibilidad del proceso, que permita capturar las características únicas de cada proyecto, potenciando la creación de iniciativas de desarrollo productivo de los grupos familiares primarios, transformando la actividad en un sistema fructífero para las comunidades [72], [77], [101]. Desarrollos como Global Reporting Initiative, así como iniciativas lideradas por el Foro Intergubernamental de Minería, han logrado definir políticas que promueven la sostenibilidad del sector minero y la reducción de la pobreza, estrategias que han sido enfocadas en más de 60 países y han sido respaldadas por un conjunto de protocolos que las compañías mineras miden e informan en las áreas de alcance comunitario, 
incluyendo el consumo de energía y emisiones, volúmenes de material de relaves, prácticas para la conservación de la biodiversidad, seguridad y salud, gestión de crisis y prevención del trabajo infantil forzado, manteniendo la continuidad y la coherencia en todas las fases de los proyectos extractivos [102]-[104].

\subsection{Perspectivas, avances y retos sociales}

La industria minera impacta las economías locales, las estructuras sociales y los valores culturales en aquellos lugares donde las comunidades dependen directamente de sus recursos naturales y de los servicios ambientales para su sustento. Estas comunidades son las más afectadas [105]. Estas comunidades están sometidas a condiciones de desplazamiento por las actividades mineras y sufren los efectos de la creación de tierras baldías a partir de minas a cielo abierto y la remoción de la capa superficial del suelo, la contaminación de agua, de aire y de suelo, además de la lixiviación ácida y de la deforestación [106]. Sin embargo, también existen acciones no siempre aplicables para restablecer el sustento de los agricultores afectados. La recuperación se puede realizar mediante una combinación de plantas perennes leñosas, cultivos anuales y pastoreo de ganado que pueden contribuir al mejoramiento del suelo en términos de estructura, carbono orgánico y estado de nutrientes, al mismo tiempo que producen ahorro en tiempos, producción de frutas y/o alimentos, madera y leña para la comunidad [107].

Los agricultores deben estar incluidos en los procesos de restauración desde las primeras etapas de planificación del proyecto para aumentar su compromiso de cumplir voluntariamente con los desafíos de recuperar tierras minadas [108]. En un estudio de caso de recuperación de tierras, realizado por AngloGold Ashanti Ltd., se mostró que el 90 \% de la comunidad participó [109]. Los principales beneficios percibidos fueron la mejora de la seguridad alimentaria mediante el aumento de la producción agrícola, las oportunidades de acceso a la tierra para grupos marginados, el aumento del valor monetario de la tierra, la mitigación de riesgos ambientales, la generación de empleo y la disminución de conflictos.

Además de los esquemas de recuperación, han surgido iniciativas como Oro Verde para abordar sus propias necesidades. Oro Verde comenzó cuando los mineros artesanales buscaron apoyo en la administración pública y establecieron vínculos con organizaciones no gubernamentales que estaban activas en la región del Chocó. Desde el año 2000, la iniciativa ha desarrollado prácticas de minería responsable certificadas, impulsando un movimiento de comercio justo en torno a la función de la minería responsable. El oro es extraído por mineros artesanales que cumplen con estrictos estándares ecológicos y estos productos se venden bajo la marca Oro Verde a los mercados en Europa y América del Norte [110]. El resultado fue un nuevo sistema de gobernanza en el que se aplicaron normativas locales y políticas nacionales, de modo que los mineros fueron reconocidos oficialmente por Fairtrade-Fairmined, una ONG internacional que opera en Colombia. Este logro ha generado reconocimiento internacional, así como beneficios económicos, ya que los mineros pueden vender su producto a precios hasta un $15 \%$ más altos que el mercado regular [111].

\section{CONCLUSIONES}

El gobierno colombiano definió el sector de la minería como un importante motor del desarrollo para la economía. Aunque su expansión durante la última década ha puesto en evidencia sus riesgos, también ha permitido algunas iniciativas de beneficio económico locales incipientes. Desde el punto de vista ambiental, con el aumento de la actividad extractiva, la 
tierra degradada aumentará significativamente. Se ha demostrado que la actividad se ha duplicado en los últimos 15 años. El aumento de las actividades de extracción artesanales no reguladas tiene el potencial de seguir generando emisiones de mercurio. Además, las tecnologías actuales que se utilizan para la extracción industrial continuarán ejerciendo presión sobre la disponibilidad de los recursos minerales debido a la alta intensidad de sus operaciones. A pesar de la proliferación de regulaciones, la informalidad en la minería de oro sigue siendo evidente, lo que conlleva a una actividad extractiva sin seguridad social, gestión ambiental o regulaciones de seguridad y salud en el trabajo, y la evasión del pago de regalías al Estado.

A pesar de la imagen negativa de la extracción de oro, el sector tiene el potencial de conferir beneficios económicos a las comunidades. Por lo tanto, es de suma importancia encontrar estrategias de supervivencia adecuadas para las áreas afectadas por la minería donde se combine beneficio económico local y practicas más sustentables que no impliquen riesgos para la comunidad. Las prácticas de recuperación deben planificarse y gestionarse bajo esquemas que incluyan la estabilización del suelo, el control de la contaminación y la restauración paisajística. Adicionalmente se deben implementar plantas de procesamiento sin mercurio, así como la creación de asociaciones entre mineros artesanales y de pequeña escala para acceder a recursos del Estado y adquirir tecnologías más limpias para el procesamiento del oro. Asimismo, deben crearse estrategias de remediación adecuadas para los humedales, suelos y ríos contaminados. Para lograr esto, un paso crucial es desarrollar marcos regulatorios efectivos dirigidos a reducir la informalidad de la minería, brindando pautas para la planificación y el diseño de planes de gestión ambiental, incluyendo normas de seguridad para la actividad y la protección de la biodiversidad. Para agregar valor a las actividades de extracción, las comunidades locales deben participar y deben considerarse sus necesidades y expectativas. Para ello es necesario crear esquemas de compensación adecuados. Además, el monitoreo de las consecuencias que las actividades mineras generan sobre la salud de las personas que viven en las áreas circundantes. Es necesario mejorar la comunicación efectiva y la transparencia entre las empresas mineras y las comunidades locales, reduciendo conflictos y promoviendo confianza, así como una comprensión más profunda de las comunidades para tomar decisiones sobre el uso de la tierra luego del cierre del proceso y participación en los diseños y esquemas de recuperación ambiental.

Todavía existe una brecha de conocimiento sobre la evaluación del ciclo de vida de los procesos de extracción. En este sentido, la cuantificación de las emisiones de gases de efecto invernadero, el consumo de agua y la carga de residuos sólidos deben medirse de manera sistemática, ya que representan una importante oportunidad de mejora del sector, incluyendo la restauración forestal, la protección de la biodiversidad y el funcionamiento de los ecosistemas de las áreas afectadas por los desechos de la minería de oro. El camino hacia una minería socialmente sólida, con esquemas de distribución de regalías, debe diseñarse de tal manera que garantice el compromiso de las instituciones locales con el desarrollo del sector y compense las menores oportunidades de sustento de la comunidad a través de la asignación eficiente de recursos mediante la implementación de planes de responsabilidad social corporativa que contribuyan a mantener el valor de la comunidad al convertir el capital financiero del desarrollo de recursos naturales. De igual forma, la defensa de los medios de sustento de las comunidades como uno de los reclamos centrales en los conflictos mineros y la implementación de mecanismos de participación apoyados en la creación de relaciones entre empresas mineras y comunidades basadas en el respeto, la inclusión y la transparencia. 


\section{AGRADECIMIENTOS}

Los autores agradecen la asistencia financiera de la Universidad Autónoma Latinoamericana.

\section{CONFLICTOS DE INTERÉS DE LOS AUTORES}

No hay conflicto de intereses que declarar.

\section{CONTRIBUCIÓN DE LOS AUTORES}

Todos los autores contribuyeron de igual manera a la compilación y análisis de la información, así como con la redacción y discusión sobre el tema. Específicamente:

Juan D. Ospina-Correa concibió la idea del artículo y se centró en la elaboración de los vínculos entre las diferentes estructuras.

Juan G. Osorio-Cachaya desarrolló la argumentación geológica para los filtros de las búsquedas iniciales y técnicas de análisis.

Ángela M. Henao-Arroyave acompañó la argumentación geológica realizada para los filtros de las búsquedas iniciales y técnicas de caracterización mineral.

Frank Sánchez-Restrepo desarrolló la argumentación geopolítica realizada para los filtros de las búsquedas iniciales.

Jim Giraldo-Builes desarrolló la argumentación socio-económica y aspectos de innovación de procesos realizada para los filtros de las búsquedas iniciales.

\section{REFERENCIAS}

[1] A. Rettberg; J. F. Ortiz-Riomalo, "Golden Opportunity, or a New Twist on the Resource-Conflict Relationship: Links Between the Drug Trade and Illegal Gold Mining in Colombia,” World Dev., vol. 84, pp. 82-96, Aug. 2016. https://doi.org/10.1016/j.worlddev.2016.03.020

[2] D. Acemoglu; C. García-Jimeno; J. A. Robinson, "Finding Eldorado: Slavery and long-run development in Colombia," J. Comp. Econ., vol. 40, no. 4, pp. 534-564, Nov. 2012. https://doi.org/10.1016/j.jce.2012.07.003

[3] M. M. Veiga; G. Angeloci-Santos; J. A. Meech, "Review of barriers to reduce mercury use in artisanal gold mining," Extractive Industries and Society, vol. 1, no. 2. pp. 351-361, Nov. 2014. https://doi.org/10.1016/j.exis.2014.03.004

[4] Ministerio de Minas y Energía, "Política minera de Colombia. Bases para la minería del futuro, "Bogotá, Julio 2016. URL

[5] Ministerio de Minas y Energía, "Boletín Estadístico de Minas y Energía 2016- 2018,” Nov. 2018. URL

[6] O. García; M. M. Veiga; P. Cordy, O. E. Suescún; J. M. Molina; M. Roeser, "Artisanal gold mining in Antioquia, Colombia: A successful case of mercury reduction,” J. Clean. Prod., vol. 90, pp. 244-252, Mar. 2015. https://doi.org/10.1016/j.jclepro.2014.11.032

[7] J. J. Hinton, Communities and Small-Scale Mining: An Integrated Review for Development Planning. 2005. $\underline{\mathrm{URL}}$

[8] N. L. Alvarez-Berrios; T. Mitchell Aide, "Global demand for gold is another threat for tropical forests," Environ. Res. Lett., vol. 10, no. 1, Jan. 2015, https://doi.org/10.1088/1748-9326/10/1/014006

[9] P. Cordy et al., "Mercury contamination from artisanal gold mining in Antioquia, Colombia: The world's highest per capita mercury pollution," Sci. Total Environ., vol. 410-411, pp. 154-160, Dec. 2011, https://doi.org/10.1016/j.scitotenv.2011.09.006

[10] S. B. Lovitz, "Scales of responsible gold mining: overcoming barriers to cleaner artisanal mining in southern Ecuador,” (Tesis de maestría), University of Vermont, 2006. URL 
[11] D. Specht; M. A. F. Ros-Tonen, "Gold, power, protest: Digital and social media and protests against largescale mining projects in Colombia," New Media Soc., vol. 19, no. 12, pp. 1907-1926, May. 2017. https://doi.org/10.1177/1461444816644567

[12] M. Fritz; J. McQuilken; N. Collins; F. Weldegiorgis, "Global trends in artisanal and small-scale mining (asm): a review of key numbers and issues." Institute for Sustainable Development, p. 81, 2018. URL

[13] P. Cordy; M. Veiga; L. Bernaudat; O. Garcia, "Successful airborne mercury reductions in Colombia," J. Clean. Prod., vol. 108, pp. 992-1001, Dec. 2015. https://doi.org/10.1016/j.jclepro.2015.06.102

[14] M. M. Veiga; B. G. Marshall, "The Colombian artisanal mining sector: Formalization is a heavy burden," Extr. Ind. Soc., vol. 6, no. 1, pp. 223-228, Jan. 2019, https://doi.org/10.1016/j.exis.2018.11.001

[15] D. M. Agudelo-Echavarría; C. Olid, F. Molina-Pérez; P. P. Vallejo-Toro; J. Garcia-Orellana, "Historical reconstruction of Small-scale gold mining activities in tropical wetland sediments in Bajo Cauca-Antioquia, Colombia," Chemosphere, vol. 254, Sep. 2020. https://doi.org/10.1016/j.chemosphere.2020.126733

[16] B. Betancur-Corredor; J. C. Loaiza-Usuga; M. Denich; C. Borgemeister, "Gold mining as a potential driver of development in Colombia: Challenges and opportunities," J. Clean. Prod., vol. 199, pp. 538-553, Oct. 2018. https://doi.org/10.1016/j.jclepro.2018.07.142

[17] Ministerio de Minas y Energía, "Minería - Ministerio de Minas y Energía," Cifras Sector Minero. 2017. URL

[18] L. Güiza Suarez; J. D. Aristizábal, "Mercury and gold mining in Colombia: A failed state,” Univ. Sci., vol. 18, no. 1, pp. 33-49, Feb. 2013, https://doi.org/10.11144/javeriana.SC18-1mgmc

[19] C. G. Ntim; J. English; J. Nwachukwu; Y. Wang, "On the efficiency of the global gold markets," Int. Rev. Financ. Anal., vol. 41, pp. 218-236, Oct. 2015. https://doi.org/10.1016/j.irfa.2015.03.013

[20] A. Martínez; T. Aguilar, "Estudio sobre los impactos socioeconómicos del sector minero en colombia: encadenamientos sectoriales," Cuad. Fedesarrollo, vol. 47, May. 2013. URL

[21] M. Walter; L. Urkidi, "Community mining consultations in Latin America (2002-2012): The contested emergence of a hybrid institution for participation," Geoforum, vol. 84, pp. 265-279, Aug. 2017. https://doi.org/10.1016/j.geoforum.2015.09.007

[22] E. Abud, "The Cambridge Economic History of Latin America (review)," Arizona J. Hisp. Cult. Stud., vol. 10, pp. 291-292, 2006, https://doi.org/10.1353/hcs.2007.0000

[23] Reporte Minero, "Sector minero aporta el 10\% del PIB nacional," Reporte Minero., Apr. 2018. URL

[24] A. Whitmore, "The emperors new clothes: Sustainable mining?," J. Clean. Prod., vol. 14, no. 3-4, pp. 309314, 2006. https://doi.org/10.1016/j.jclepro.2004.10.005

[25] K. W. Butzer, "Collapse, environment, and society," Proceedings of the National Academy of Sciences, vol. 109, no. 10, pp. 3632-3639, Mar. 2012. https://doi.org/10.1073/pnas.1114845109

[26] Investing.com, "Gold Futures Historical Prices," investing.com, 2020. URL

[27] Investing.com, "Nickel Futures Historical Prices," investing.com, Accessed: Aug. 15, 2020. URL

[28] Investing.com, "Platinum Futures Historical Prices," 2020. Accessed: Aug. 15, 2020. URL

[29] J. Horner; A. Naranjo; J. Weil, "Digital data acquisition and 3D structural modelling for mining and civil engineering - the La Colosa gold mining project, Colombia," Geomech. und Tunnelbau, vol. 9, no. 1, pp. 5257, Feb. 2016. https://doi.org/10.1002/geot.201500046

[30] A. L. R. Madrid; T. Bissig; C. J. R. Hart; L. C. M. Figueroa, "Late pliocene high-sulfidation epithermal gold mineralization at the la bodega and la mascota deposits, northeastern cordillera of Colombia," Econ. Geol., vol. 112, no. 2, pp. 347-374, 2017. https://doi.org/10.2113/econgeo.112.2.347

[31] E. Naranjo Sierra; M. Alvaran Echeverri; E. Zapata Cardona, "Análisis metalogenético preliminar del depósito vetiforme en la mina La Ye, Antoquia, Colombia: características geológicas , isotópicas y estructurales," Rev. Mex. Ciencias Geológicas, vol. 33, mun. 3, pp. 316-328, 2016. http://www.scielo.org.mx/scielo.php?script=sci_arttext\&pid=S1026-87742016000300316

[32] C. Fuller; R. Bygness, "Colombia gold mine MSE project," 2014. URL

[33] R. B. Gibson, "Sustainability assessment and conflict resolution: Reaching agreement to proceed with the Voisey's Bay nickel mine," J. Clean. Prod., vol. 14, no. 3-4, pp. 334-348, 2006. https://doi.org/10.1016/j.jclepro.2004.07.007

[34] J. Marrugo-Negrete; J. Pinedo-Hernández; S. Díez, "Assessment of heavy metal pollution, spatial distribution and origin in agricultural soils along the Sinú River Basin, Colombia,” Environ. Res., vol. 154, pp. 380-388, Apr. 2017. https://doi.org/10.1016/j.envres.2017.01.021

[35] C. Salazar-Camacho; M. Salas-Moreno; S. Marrugo-Madrid; J. Marrugo-Negrete; S. Díez, "Dietary human exposure to mercury in two artisanal small-scale gold mining communities of northwestern Colombia," Environ. Int., vol. 107, pp. 47-54, Oct. 2017. https://doi.org/10.1016/j.envint.2017.06.011

[36] B. Betancur-Corredor; J. C. Loaiza-Usuga; M. Denich; C. Borgemeister, "Gold mining as a potential driver of development in Colombia: Challenges and opportunities," Journal of Cleaner Production, vol. 199. pp. 538-553, Oct. 2018. https://doi.org/10.1016/j.jclepro.2018.07.142 
[37] L. A. Rodríguez-Villamizar; D. C. Jaimes; A. Manquián-Tejos; L. H. Sánchez, "Irregularidad menstrual y exposición a mercurio en la minería artesanal del oro en Colombia," Biomedica, vol. 35, no. Suplemento 2, pp. 38-45, Aug. 2015. https://doi.org/10.7705/biomedica.v35i0.2442

[38] P. H. Raven, "Science, sustainability, and the human prospect," vol. 297, no. 5583, pp. 954-958. https://doi.org/10.1126/science.297.5583.954

[39] N. Bustamante; N. Danoucaras; N. McIntyre, J. C. Díaz-Martínez; O. J. Restrepo-Baena, "Review of improving the water management for the informal gold mining in Colombia," Rev. Fac. Ing., no. 79, pp. 174-184, Jun. 2016. https://doi.org/10.17533/udea.redin.n79a16

[40] K. Hearn, "Mining Trouble," E - Environ. Mag., vol. 17, no. 1, pp. 10-12, Jan. 2006. URL

[41] M. C. Prada Fonseca; G. Caicedo Pineda; M. Márquez Godoy, "Comparación del potencial oxidativo de Acidithiobacillus ferrooxidans, en un proceso de biodesulfurización de carbón,” Rev. Colomb. Biotecnol., vol. 18, no. 1, 2016. https://doi.org/10.15446/rev.colomb.biote.v18n1.50274

[42] J. A. McNeish, "A vote to derail extraction: popular consultation and resource sovereignty in Tolima, Colombia," Third World Q., vol. 38, no. 5, pp. 1128-1145, Feb. 2017. https://doi.org/10.1080/01436597.2017.1283980

[43] L. Güiza Suárez, "Juridical Perspective of Environmental Impact on Water Resources Caused by Mining in Colombia," Opinión Jurídica, vol. 10, no. SPE, pp. 123-140, Dec. 2011. URL

[44] R. J. Batterham, "The mine of the future - Even more sustainable," Miner. Eng., vol. 107, pp. 2-7, 2017. https://doi.org/10.1016/j.mineng.2016.11.001

[45] T. Norgate; N. Haque, "Using life cycle assessment to evaluate some environmental impacts of gold production," J. Clean. Prod., vol. 29-30, pp. 53-63, Jul. 2012, https://doi.org/10.1016/j.jclepro.2012.01.042

[46] H. Snyder, "Literature review as a research methodology: An overview and guidelines," J. Bus. Res., vol. 104, pp. 333-339, Nov. 2019. https://doi.org/10.1016/j.jbusres.2019.07.039

[47] S. J. Gallagher, "Book and Media Reviews: Conducting Research Literature Reviews: From the Internet to Paper," New Horizons Adult Educ. Hum. Resour. Dev., vol. 20, no. 4, pp. 41-43, Jan. 2013. https://doi.org/10.1002/nha3.10270

[48] J. Białkowski; M. T. Bohl; P. M. Stephan; T. P. Wisniewski, "The gold price in times of crisis," Int. Rev. Financ. Anal., vol. 41, pp. 329-339, Oct. 2015. https://doi.org/10.1016/j.irfa.2014.07.001

[49] N. Idrobo; D. Mejía; A. M. Tribin, "Illegal gold mining and violence in Colombia," Peace Econ. Peace Sci. Public Policy, vol. 20, no. 1, pp. 83-111, 2014. https://doi.org/10.1515/peps-2013-0053

[50] C. Ochoa, "Generating conflict: Gold, water and vulnerable communities in the Colombian Highlands," in Natural Resources and Sustainable Development: International Economic Law Perspectives, 2017, pp. 142164.

[51] World Gold Council, Socio-Economic Impact of Mining Gold. 2015. URL

[52] E. G. Egidarev; E. A. Simonov, "Assessment of the environmental effect of placer gold mining in the Amur river basin," Water Resour., vol. 42, no. 7, pp. 897-908, Dec. 2015. https://doi.org/10.1134/S0097807815070039

[53] J. J. Barry et al., "The Mineral Industries of Latin America and Canada," U.S. Geological survey minerals yearbook, U.S. Department of the Interior U.S. Geological Survey, 2015. URL

[54] J. A. Villa; C. Tobón, "Modeling hydrologic dynamics of a created wetland, Colombia," Ecol. Eng., vol. 40, pp. 173-182, Mar. 2012. https://doi.org/10.1016/j.ecoleng.2011.12.005

[55] S. A. Shlyakhov; S. V. Osipov, "Technogenic surface formations in the areas of placer gold mining (the Bureya Upland, Far East)," Eurasian Soil Sci., vol. 37, no. 10, pp. 1106-1114, 2004. URL

[56] Oficina de las naciones Unidas contra la droga y el delito (UNODC); Ministerio de Minas y Energía, "Colombia Explotación de oro de aluvión. Evidencias a partir de percepción remota 2018.," Nov. 2019. URL

[57] Oficina de las naciones Unidas contra la droga y el delito UNODC; Gobierno de Colombia, "Monitoreo de territorios afectados por cultivos ilícitos 2016," Jul. 2017. URL

[58] M. Wahsha; M. M. D. Al-Rshaidat, "Potentially harmful elements in abandoned mine waste," in PHEs, Environment and Human Health, 2014, pp. 199-220. https://doi.org/10.1007/978-94-017-8965-3_5

[59] A. Calderon; J. D. Harris; P. A. Kirsch, "Health interventions used by major resource companies operating in Colombia," Resour. Policy, vol. 47, pp. 187-197, Mar. 2016. https://doi.org/10.1016/j.resourpol.2015.02.003

[60] S. Siegel, "Community without solidarity: Mercury pollution from smallscale mining and Colombia's crisis of authority," Community Dev. J., vol. 48, no. 3, pp. 451-465, Jul. 2013. https://doi.org/10.1093/cdj/bst027

[61] M. M. Veiga; G. Angeloci; M. Hitch; P. Colon Velasquez-Lopez, "Processing centres in artisanal gold mining," J. Clean. Prod., vol. 64, pp. 535-544, 2014. https://doi.org/10.1016/j.jclepro.2013.08.015

[62] MinMinas, "Plan nacional de desarrollo minero con horizonte a 2025 Minería responsable con el territorio," SIMCO - Sistema de Información Minero Colombiano, 2008. URL 
[63] A. Saldarriaga-Isaza; C. Villegas-Palacio; S. Arango, "Phasing out mercury through collective action in artisanal gold mining: Evidence from a framed field experiment," Ecol. Econ., vol. 120, pp. 406-415. Dec. 2015, https://doi.org/10.1016/j.ecolecon.2015.04.004

[64] J. Pinedo-Hernández; J. Marrugo-Negrete; S. Díez, "Speciation and bioavailability of mercury in sediments impacted by gold mining in Colombia," Chemosphere, vol. 119, pp. 1289-1295, Jan. 2015. https://doi.org/10.1016/j.chemosphere.2014.09.044

[65] J. Marrugo-Negrete; J. Pinedo-Hernández; S. Díez, "Geochemistry of mercury in tropical swamps impacted by gold mining," Chemosphere, vol. 134, pp. 44-51, Sep. 2015. https://doi.org/10.1016/j.chemosphere.2015.03.012

[66] M. Aguilera, "La Mojana: riqueza natural y potencial económico," banco la república, no. 48, p. 73, 2004. URL

[67] E. De Miguel; D. Clavijo; M. F. Ortega; A. Gómez, "Probabilistic meta-analysis of risk from the exposure to $\mathrm{Hg}$ in artisanal gold mining communities in Colombia," Chemosphere, vol. 108, pp. 183-189, Aug. 2014. https://doi.org/10.1016/j.chemosphere.2014.01.035

[68] S. Alvarez; A. M. Jessick; J. A. Palacio; A. S. Kolok, "Methylmercury concentrations in six fish species from two Colombian rivers," Bull. Environ. Contam. Toxicol., vol. 88, no. 1, pp. 65-68, 2012. https://doi.org/10.1007/s00128-011-0458-x

[69] K. S. Andam; P. J. Ferraro; A. Pfaff; G. A. Sanchez-Azofeifa; J. A. Robalino, "Measuring the effectiveness of protected area networks in reducing deforestation,” Proc. Natl. Acad. Sci., vol. 105, no. 42, pp. 16089-16094, 2008. https://doi.org/10.1073/pnas.0800437105

[70] W. C. Clark, "Sustainability science: A room of its own," Proc. Natl. Acad. Sci, vol. 104, no. 6. pp. 17371738, United States of America, 2007. https://doi.org/10.1073/pnas.0611291104

[71] J. Liu et al., "Complexity of coupled human and natural systems," Science, vol. 317, no. 5844. pp. 15131516, Sep. 2007, https://doi.org/10.1126/science.1144004

[72] R. W. Kates et al., "Sustainability science," Science, vol. 292, no. 5517. pp. 641-642, Apr. 2001. https://doi.org/10.1126/science.1059386

[73] S. R. Carpenter et al., "Millennium ecosystem assessment: Research needs," Science, vol. 314, no. 5797. pp. 257-258, Oct. 2006. https://doi.org/10.1126/science.1131946

[74] G. C. Daily; P. A. Matson, "Ecosystem services: From theory to implementation," Proc. Natl. Acad. Sci, vol. 105, no. 28. pp. 9455-9456, Jul. 2008. https://doi.org/10.1073/pnas.0804960105

[75] B. L. Turner, E. F. Lambin; A. Reenberg, "The emergence of land change science for global environmental change and sustainability," Proc. Natl. Acad. Sci., vol. 104, no. 52. pp. 20666-20671, 2007. https://doi.org/10.1073/pnas.0704119104

[76] R. Hueting; L. Reijnders, "Broad sustainability contra sustainability: The proper construction of sustainability indicators," Ecol. Econ., vol. 50, no. 3-4, pp. 249-260, Oct. 2004. https://doi.org/10.1016/j.ecolecon.2004.03.031

[77] W. V. Reid et al., "Earth system science for global sustainability: Grand challenges," Science, vol. 330 , no. 6006. pp. 916-917, Nov. 2010. https://doi.org/10.1126/science.1196263

[78] N. C. Banning; C. D. Grant; D. L. Jones; D. V. Murphy, "Recovery of soil organic matter, organic matter turnover and nitrogen cycling in a post-mining forest rehabilitation chronosequence," Soil Biol. Biochem., vol. 40, no. 8, pp. 2021-2031, Aug. 2008. https://doi.org/10.1016/j.soilbio.2008.04.010

[79] V. Sheoran; A. Sheoran; P. Poonia, "Soil Reclamation of Abandoned Mine Land by Revegetation: A Review," Int. J. Soil, Sediment Water, vol. 3, no. 2, 2010. URL

[80] J. C. Barliza; J. D. L. Peláez, "Litterfall and nutrient dynamics in Acacia mangium (Mimosaceae) forest plantations of Antioquia, Colombia," Acta Biol. Colomb., vol. 15, no. 2, pp. 289-308, May. 2010. URL

[81] S. F. Gould, "Comparison of Post-mining Rehabilitation with Reference Ecosystems in Monsoonal Eucalypt Woodlands, Northern Australia," Restor. Ecol., vol. 20, no. 2, pp. 250-259, Mar. 2012, https://doi.org/10.1111/j.1526-100X.2010.00757.x

[82] A. E. Lewis, "Review of metal sulphide precipitation," Hydrometallurgy, vol. 104, no. 2, pp. 222-234, Sep. 2010. https://doi.org/10.1016/j.hydromet.2010.06.010

[83] C. P. Brough; R. Warrender; R. J. Bowell; A. Barnes; A. Parbhakar-Fox, "The process mineralogy of mine wastes," Miner. Eng., vol. 52, pp. 125-135, Oct. 2013, https://doi.org/10.1016/j.mineng.2013.05.003

[84] E. Higgs, "The two-culture problem: Ecological restoration and the integration of knowledge," Restor. Ecol., vol. 13, no. 1, pp. 159-164, Mar. 2005. https://doi.org/10.1111/j.1526-100X.2005.00020.x

[85] D. S. Kim; K. Choi, "Global trends in mercury management," J. Prev. Med. Public Heal., vol. 45, no. 6, pp. 364-373, Nov. 2012. https://doi.org/10.3961/jpmph.2012.45.6.364

[86] M. A. Coulter, "Minamata Convention on Mercury," Int. Leg. Mater., vol. 55, no. 3, pp. 582-616, Jun. 2016. https://doi.org/10.5305/intelegamate.55.3.0582 
[87] Programa de las Naciones Unidas para el Medio Ambiente, "Elaboración de un plan de acción nacional para reducir y, cuando sea posible, eliminar el uso del mercurio en la extracción de oro artesanal y en pequeña escala," 2015-2020. URL

[88] H. Ali; E. Khan; M. A. Sajad, "Phytoremediation of heavy metals-Concepts and applications," Chemosphere, vol. 91, no. 7. pp. 869-881, May. 2013, https://doi.org/10.1016/j.chemosphere.2013.01.075

[89] A. Bhargava; F. F. Carmona; M. Bhargava; S. Srivastava, "Approaches for enhanced phytoextraction of heavy metals," Journal of Environmental Management, vol. 105. pp. 103-120, Aug. 2012. https://doi.org/10.1016/j.jenvman.2012.04.002

[90] J. Marrugo-Negrete; J. Durango-Hernández; J. Pinedo-Hernández; G. Enamorado-Montes; S. Díez, "Mercury uptake and effects on growth in Jatropha curcas," J. Environ. Sci., vol. 48, pp. 120-125, Oct. 2016. https://doi.org/10.1016/j.jes.2015.10.036

[91] B. V. Tangahu; S. R. Sheikh Abdullah; H. Basri; M. Idris; N. Anuar; M. Mukhlisin, "A review on heavy metals (As, $\mathrm{Pb}$, and $\mathrm{Hg}$ ) uptake by plants through phytoremediation,” J. Chem. Eng.,Aug. 2011. https://doi.org/10.1155/2011/939161

[92] S. Yachi; M. Loreau, "Biodiversity and ecosystem productivity in a fluctuating environment: The insurance hypothesis," Proc. Natl. Acad. Sci. U. S. A., vol. 96, no. 4, pp. 1463-1468, 1999. https://doi.org/10.1073/pnas.96.4.1463

[93] B. L. Turner; E. F. Lambin; A. Reenberg, "The emergence of land change science for global environmental change and sustainability," Proc. Natl. Acad. Sci., vol. 104, no. 52. pp. 20666-2067, 2007. https://doi.org/10.1073/pnas.0704119104

[94] J. Seccatore; G. de Tomi; M. Veiga, "Efficiency as a road to sustainability in small scale mining," Mater. Sci. Forum, vol. 805, pp. 395-402, Sep. 2014. https://doi.org/10.4028/www.scientific.net/MSF.805.395

[95] S. Nasirov; C. A. Agostini, "Mining experts' perspectives on the determinants of solar technologies adoption in the Chilean mining industry," Renew. Sustain. Energy Rev., vol. 95, pp. 194-202, Nov. 2018. https://doi.org/10.1016/j.rser.2018.07.038

[96] F. S. Chapin et al., "Policy strategies to address sustainability of Alaskan boreal forests in response to a directionally changing climate," Proc. Natl. Acad. Sci. U. S. A., vol. 103, no. 45, pp. 16637-16643, Aug. 2006. https://doi.org/10.1073/pnas.0606955103

[97] D. L. Alonso; R. Pérez; C. K. Y. A. Okio; E. Castillo, "Assessment of mining activity on arsenic contamination in surface water and sediments in southwestern area of Santurbán paramo, Colombia," J. Environ. Manage., vol. 264, Jun. 2020. https://doi.org/10.1016/j.jenvman.2020.110478

[98] B. Duarte-Abadía; R. Boelens, "Disputes over territorial boundaries and diverging valuation languages: the Santurban hydrosocial highlands territory in Colombia,” Water Int., vol. 41, no. 1, pp. 15-36, Jan. 2016. https://doi.org/10.1080/02508060.2016.1117271

[99] Ministerio de Ambiente y Desarrollo Sostenible, "Guía técnica para la formulación de planes de ordenación y manejo de cuencuas hidrográficas, 2014. URL

[100] A. E. Fordham; G. M. Robinson; B. D. Blackwell, "Corporate social responsibility in resource companies Opportunities for developing positive benefits and lasting legacies," Resour. Policy, vol. 52, pp. 366-376, Jun. 2017. https://doi.org/10.1016/j.resourpol.2017.04.009

[101] J. J. Hinton; M. M. Veiga; A. T. C. Veiga, "Clean artisanal gold mining: A utopian approach?," Journal of Cleaner Production, vol. 11, no. 2. pp. 99-115, Mar. 2003. https://doi.org/10.1016/S0959-6526(02)00031-8

[102] A. Fonseca; M. L. McAllister; P. Fitzpatrick, "Sustainability reporting among mining corporations: A constructive critique of the GRI approach," Journal of Cleaner Production, vol. 84, no. 1. pp. 70-83, Dec. 2014. https://doi.org/10.1016/j.jclepro.2012.11.050

[103] G. M. Mudd, "Global trends in gold mining: Towards quantifying environmental and resource sustainability," Resour. Policy, vol. 32, no. 1-2, pp. 42-56, Jun. 2007. https://doi.org/10.1016/j.resourpol.2007.05.002

[104] M. Tost; M. Hitch; V. Chandurkar; P. Moser; S. Feiel, "The state of environmental sustainability considerations in mining," J. Clean. Prod., vol. 182, pp. 969-977, May. 2018. https://doi.org/10.1016/j.jclepro.2018.02.051

[105] J. Lyytimäki; L. Peltonen, "Mining through controversies: Public perceptions and the legitimacy of a planned gold mine near a tourist destination," Land use policy, vol. 54, pp. 479-486, Jul. 2016, https://doi.org/10.1016/j.landusepol.2016.03.004

[106] UNDP, "Human Development Indices and Indicators. 2018 Statistical Update,” 2018. URL

[107] N. Sharma; B. Bohra; N. Pragya; R. Ciannella; P. Dobie; S. Lehmann, "Bioenergy from agroforestry can lead to improved food security, climate change, soil quality, and rural development," Food and Energy Security, vol. 5, no. 3. pp. 165-183, Aug. 2016, https://doi.org/10.1002/fes3.87

[108] R. I. Maczkowiack; C. S. Smith; G. J. Slaughter; D. R. Mulligan; D. C. Cameron, "Grazing as a post-mining land use: A conceptual model of the risk factors," Agric. Syst., vol. 109, pp. 76-89, Jun. 2012. https://doi.org/10.1016/j.agsy.2012.03.002 
[109] E. N. Tetteh; V. Logah; K. Twum-Ampofo; S. T. Partey, "Effect of duration of reclamation on soil quality indicators of a surface - Mined acid forest Oxisol in South - Western Ghana," West African J. Appl. Ecol., vol. 23, no. 2, pp. 63-72, 2015. URL

[110] N. A Cano; H. I. Velasquez Arredondo, "Sustainability assessment by emergy approach: Gold mining extraction in Colombia," in Environmental Division 2018 - Core Programming Area at the 2018 AIChE Annual Meeting, 2018. URL

[111] M. Sarmiento; H. Ayala; A. Urán, B. Giraldo; J. Perea; A. Mosquera, "Legitimidad e innovación en la minería: el caso del Programa Oro Verde," Let. Verdes, Rev. Latinoam. Estud. Socioambientales, Sep. 2013. https://doi.org/10.17141/letrasverdes.14.2013.1005 